\title{
miR-663 promotes NPC cell proliferation by directly targeting CDKN2A
}

\author{
SHAOQIANG LIANG, NING ZHANG, YANMING DENG, LUSI CHEN, YANG ZHANG, \\ ZHENHE ZHENG, WEIJUN LUO, ZHIQIAN LV, SHAOEN LI and TAO XU
}

Department of Radiotherapy, Tumor Hospital of First People's Hospital of Foshan, Foshan, Guangdong 528041, P.R. China

Received June 4, 2016; Accepted May 22, 2017

DOI: $10.3892 / \mathrm{mmr} .2017 .7129$

\begin{abstract}
MicroRNAs (miRs) act as important regulators during the development and progression of human cancer; however, the regulatory mechanism of miR-663 in nasopharyngeal carcinoma (NPC) remains unclear. The present study demonstrated that serum miR-663 levels were significantly increased in patients with NPC compared with healthy controls. In addition, the serum levels of miR-663 were associated with the grade, lymph node metastasis and clinical stage of NPC. The expression of miR-663 was increased in NPC C666-1 cells, compared with normal nasopharyngeal epithelial NP69 cells. The knockdown of miR-663 markedly decreased the proliferation of C666-1 cells through the induction of cell cycle arrest at the G1 stage. Cyclin-dependent kinase inhibitor 2A (CDKN2A) was hypothesized to be a putative target of miR-663. Further investigation confirmed that miR-663 was able to directly bind to the 3' untranslated region of CDKN2A mRNA, and to negatively regulate CDKN2A protein expression in C666-1 cells. Inhibition of CDKN2A expression attenuated the suppressive effects of miR-663 knockdown on the proliferation and cell cycle progression of C666-1 cells. In addition, it was observed that the mRNA and protein levels of CDKN2A were decreased in C666-1 cells compared with NP69 cells. In conclusion, the results of the present study demonstrated that miR-663 promoted the proliferation and cell cycle progression of NPC cells by directly targeting CDKN2A, suggesting that miR-663 may become a potential therapeutic target for the treatment of NPC.
\end{abstract}

\section{Introduction}

Nasopharyngeal carcinoma (NPC) is a common type of head and neck cancer in Southeast Asia, particularly in southern

Correspondence to: Dr Ning Zhang, Department of Radiotherapy, Tumor Hospital of First People's Hospital of Foshan, 81 North Lingnan Avenue, Foshan, Guangdong 528041, P.R. China

E-mail: foshanzhangning@sina.com

Key words: nasopharyngeal carcinoma, microRNA-663, cell proliferation, cell cycle, cyclin-dependent kinase inhibitor $2 \mathrm{~A}$
China (1). It has been previously demonstrated that genetic susceptibility and Epstein-Barr virus (EBV) infection are associated with NPC development (1-3). No obvious clinical manifestation is observed at the early stages of NPC, and the location of the tumor is not clearly visible; therefore, NPC is difficult to diagnose and the prognosis of the advanced disease is poor (4,5). Further investigation to identify effective molecular targets for NPC treatment is required.

MicroRNAs (miRs), a class of small non-coding RNAs, are able to negatively regulate gene expression by inducing mRNA degradation or suppressing protein translation, through direct binding to the 3' untranslated regions (3'UTRs) of their target mRNAs (6). It has been previously reported that miRs act as regulators of cell survival, differentiation, proliferation, cell cycle progression and tumorigenesis $(7,8)$. By mediating the expression of oncogenes or tumor suppressors, a number of miRs, including miR-663, serve suppressive or promotive roles in the development and progression of human cancer, including NPC $(9,10)$. For example, miR-218 suppresses NPC progression through inhibition of survivin and the slit homolog 2 protein-roundabout homolog 1 signaling pathway (11). miR-214 promotes tumorigenesis by targeting lactotransferrin in NPC (12). miR-663 was observed to be markedly downregulated in gastric cancer (13), and upregulated in castration-resistant prostate cancer (14). Previously, miR-663 was reported to serve a promotive role in NPC cell proliferation in vitro and NPC growth in vivo (15). However, the regulatory mechanism of miR-663 in NPC cell proliferation remains unclear.

Cyclin-dependent kinase inhibitor 2A (CDKN2A), also termed P16, is an important tumor suppressor (16). CDKN2A functions as an inhibitor of CDK4, and therefore serves a role in cell cycle phase G1 control (17). In addition, it may interact with, and sequester, E3 ubiquitin-protein ligase MDM2, a protein associated with the degradation of p53 (18). Therefore, CDKN2A is a stabilizer of the tumor suppressor p53 (18). Dysregulation of CDKN2A has been observed in various types of human cancer, including NPC (19-21). However, the precise mechanism underlying CDKN2A expression in NPC remains unknown.

Therefore, the present study aimed to investigate the expression and clinical significance of miR-663 in NPC, in addition to the regulatory mechanism of miR-663 in NPC cell viability, associated with CDKN2A. 


\section{Materials and methods}

Serum sample collection. The present study was approved by the Ethics Committee of the Tumor Hospital of First People's Hospital of Foshan (Foshan, China). A total of 74 serum samples from patients with NPC and 27 from healthy controls were obtained. The clinical characteristics of the patients with NPC are summarized in Table I (22). All of the participants gave written informed consent. Prior to blood sample collection, none of the patients had received any treatment. Serum was isolated from $10 \mathrm{ml}$ blood by centrifuging at $1,000 \mathrm{x} \mathrm{g}$ at room temperature for $5 \mathrm{~min}$, and subsequently at $12,000 \mathrm{xg}$ at $4^{\circ} \mathrm{C}$ for $5 \mathrm{~min}$. The samples were stored at $-80^{\circ} \mathrm{C}$ prior to usage.

Cell culture. NPC C666-1 cells and the normal nasopharyngeal epithelial cell line NP69 were obtained from the Type Culture Collection of the Chinese Academy of Sciences (Shanghai, China). Cells were cultured in Dulbecco's modified Eagle's medium (DMEM; Thermo Fisher Scientific, Inc., Waltham, MA, USA) supplemented with $10 \%$ fetal bovine serum (Gibco; Thermo Fisher Scientific, Inc.) in a $37^{\circ} \mathrm{C}$ humidified atmosphere containing $5 \% \mathrm{CO}_{2}$.

Cell transfection. For transfection, C666-1 cells were cultured to $70 \%$ confluence and transfected with $100 \mathrm{nM}$ pcDNA3.1-CDKN2A open reading frame plasmid (Yearthbio, Changsha, China), pcDNA3.1 blank vector (both Promega Corporation, Madison, WI, USA), miR-663 inhibitor (GeneCopoeia, Inc., Rockville, MD, USA), negative control (NC) inhibitor (GeneCopoeia, Inc.), miR-663 mimic (GeneCopoeia, Inc.), or scramble miR mimic (GeneCopoeia, Inc.) using Lipofectamine 2000 (Thermo Fisher Scientific, Inc.), for $48 \mathrm{~h}$ according to the manufacturer's protocol. The following links demonstrate the miR-663 mimic and NC sequences, respectively: http://www.fulengen $. \mathrm{com} /$ product $/ \mathrm{search} 2 /$ ?s=miR-663\&search_type $=1 \&$ submit $=$ Submit and http://www.fulengen.com/product/reportervector-controls.

Reverse transcription-quantitative polymerase chain reaction ( $R T-q P C R$ ) analysis. Total RNA was extracted from cells using TRIzol Reagent (Thermo Fisher Scientific, Inc.), which was converted to cDNA using an RevertAid Reverse Transcription kit (Thermo Fisher Scientific, Inc.), according to the manufacturer's protocol. The miR-663 expression levels were examined with qPCR using PrimeScript ${ }^{\circledR}$ miRNA RT-PCR kit (Takara Biotechnology Co., Ltd., Dalian, China) on an ABI 7500 thermocycler (Thermo Fisher Scientific, Inc.). U6 was used as an internal reference. The mRNA expression was detected by qPCR using the standard SYBR-Green RT-PCR kit (Takara Biotechnology Co., Ltd.). GAPDH was used as an internal reference. The specific primer pairs were as follows: CDKN2A sense, 5'-GATCCAGGTGGGTAGAAG GTC-3' and antisense, 5'-CCCCTGCAAACTTCGTCCT-3'; GAPDH sense, 5'-ACAACTTTGGTATCGTGGAAGG-3' and antisense, 5'-GCCATCACGCCACAGTTTC-3'. The reaction conditions were $95^{\circ} \mathrm{C}$ for $5 \mathrm{~min}$, followed by 40 cycles of denaturation at $95^{\circ} \mathrm{C}$ for $15 \mathrm{sec}$ and an annealing/elongation step at $60^{\circ} \mathrm{C}$ for $30 \mathrm{sec}$. The relative expression was analyzed using the $2^{-\Delta \Delta C q} \operatorname{method}(23)$.
Western blotting. Cells were solubilized in cold radioimmunoprecipitation assay lysis buffer (Thermo Fisher Scientific, Inc.) to extract the total protein, which was quantified using a bicinchoninic acid assay (Pierce; Thermo Fisher Scientific, Inc.) and $50 \mu \mathrm{g}$ protein was separated using SDS-PAGE on a $10 \%$ gel (Pierce; Thermo Fisher Scientific, Inc.), and transferred onto a polyvinylidene difluoride membrane (Thermo Fisher Scientific, Inc.). The membrane was blocked with 5\% non-fat milk in DMEM for $3 \mathrm{~h}$ at room temperature, followed by a rabbit anti-CDKN2A monoclonal antibody (1:100; catalog no. ab108349; Abcam, Cambridge, UK) or rabbit anti-GAPDH monoclonal antibody (1:100; catalog no. ab181602; Abcam) at room temperature for $3 \mathrm{~h}$. Following washing with PBST for 15 min, the membrane was incubated with the goat anti-rabbit secondary antibody (1:1,000; catalog no. ab6721; Abcam) at room temperature for $1 \mathrm{~h}$. Chemiluminescent detection was conducted by using an enhanced chemiluminescence kit (Pierce; Thermo Fisher Scientific, Inc.). The protein expression was analyzed using Image-Pro plus software (version 6.0; Media Cybernetics, Inc., Rockville, MD, USA), represented as the density ratio vs. GAPDH.

Cell viability analysis. C666-1 cells $\left(5 \times 10^{4}\right)$ were cultured in a 96-well plate, each well containing $100 \mu \mathrm{l}$ fresh serum-free medium with $0.5 \mathrm{~g} / 1$ MTT (Sigma-Aldrich; Merck KGaA, Darmstadt, Germany). Following incubation at $37^{\circ} \mathrm{C}$ for $12,24,48$ and $72 \mathrm{~h}$, the medium was removed by aspiration and $50 \mu \mathrm{l}$ dimethyl sulfoxide (Sigma-Aldrich; Merck KGaA) was added to each well. Following incubation at $37^{\circ} \mathrm{C}$ for a further $10 \mathrm{~min}$, the absorbance at $570 \mathrm{~nm}$ of each sample was measured using a plate reader (Tecan Infinite M200; Tecan Group Ltd., Männedorf, Switzerland).

Cell cycle distribution analysis. C666-1 cells $\left(1 \times 10^{6}\right)$ were washed twice in Dulbecco's PBS, resuspended in 70\% ethanol and fixed overnight at $-20^{\circ} \mathrm{C}$. Subsequently, cells were washed twice in PBS with 3\% bovine serum albumin (BSA, Sigma-Aldrich; Merck KGaA) and incubated for $30 \mathrm{~min}$ at room temperature in propidium iodide (PI) staining buffer containing 3\% BSA, $40 \mu \mathrm{g} / \mathrm{ml} \mathrm{PI}$, and $0.2 \mathrm{mg} / \mathrm{ml} \mathrm{RNase}$ in PBS. DNA content was determined using flow cytometry (FACSCalibur; BD Biosciences, Franklin Lake, NJ, USA) and FACSComp software version 1.0 (BD Biosciences).

Bioinformatic prediction. Targetscan software (www .targetscan.org) was used to predict the putative target genes of miR-663.

Dual luciferase reporter assay. The wild type (WT) or mutant (MT) CDKN2A 3'-UTRs were constructed using PCR and the Quick-Change Site-Directed Mutagenesis kit (Stratagene; Agilent Technologies, Inc., Santa Clara, CA, USA), which was subsequently inserted into the multiple cloning site in the psiCHECK $^{\mathrm{TM}}$ vector (Promega Corporation). C666-1 cells were cultured to $70 \%$ confluence in a 24 -well plate, and co-transfected with $100 \mathrm{ng}$ WT-CDKN2A or MT-CDKN2A vector, plus $50 \mathrm{nM}$ miR-663 mimics or scramble miR using Lipofectamine ${ }^{\circledR} 2000$. The dual luciferase reporter assay system (Promega Corporation) was used to determine the 
luciferase activities $48 \mathrm{~h}$ post-transfection. Renilla luciferase activity was normalized to firefly luciferase activity.

Statistical analysis. Data are expressed as the mean \pm standard deviation. Comparisons between groups were analyzed using Student's t test or one-way analysis of variance followed by the Tukey's post-hoc test. The association between serum miR-663 levels and the clinical characteristics of patients with NPC was analyzed using a $\chi^{2}$ test. Statistical analysis was performed using SPSS (version 19.0; IBM Corp., Armonk, $\mathrm{NY}$, USA). $\mathrm{P}<0.05$ was considered to indicate a statistically significant difference.

\section{Results}

Serum levels of miR-663 are upregulated in NPC. The serum levels of miR-663 were detected in patients with NPC and healthy controls. RT-qPCR analysis demonstrated that the serum miR-663 levels were significantly increased in patients with NPC compared with healthy controls (Fig. 1).

Serum miR-663 levels were significantly associated with grade, clinical stage and lymph node metastasis in NPC. The association between the serum miR-663 levels and clinical characteristics in patient with NPC was analyzed. Using the mean level of serum miR-663 as a threshold, the patients with NPC recruited for the present study were divided into two groups, a high serum miR-663 expression group and a low serum miR-663 expression group. It was observed that the serum miR-663 levels were significantly associated with grade, clinical stage and lymph node metastasis (Table I). However, no association was observed between the serum miR-663 levels and age, gender, tumor stage, distant metastasis or EBV infection (Table I). Therefore, increased expression of miR-663 may promote the malignant progression of NPC.

Inhibition of miR-663 reduces the proliferation of C666-1 cells and induces cell cycle arrest at G1. In order to further confirm the results detailed above, the expression of miR-663 in NPC C666-1 cells was examined, and a normal nasopharyngeal epithelial cell line, NP69, was used as a control. As demonstrated in Fig. 2A, miR-663 was upregulated in NPC cells compared with NP69 cells.

The regulatory role of miR-663 in NPC cell viability was subsequently investigated. As miR-663 was significantly upregulated in the serum of patients with NPC patients, in addition to NPC cells, C666-1 cells were transfected with a miR-663 inhibitor to knock down its expression. An NC non-specific inhibitor was additionally used. Transfection with the miR-663 inhibitor significantly decreased the miR-663 levels in C666-1 cells, compared with the control group (Fig. 2B). However, transfection with the NC inhibitor did not affect the miR-663 levels in C666-1 cells (Fig. 2B).

An MTT assay was conducted to examine cell proliferation. The results of the present study demonstrated that the knockdown of miR-663 significantly decreased the proliferation of C666-1 cells, compared with the control group (Fig. 2C). Cell cycle progression serves an important role in cell proliferation. As presented in Fig. 2D, inhibition of miR-663 led to a significant arrest at the G1 stage in C666-1 cells. The results of the

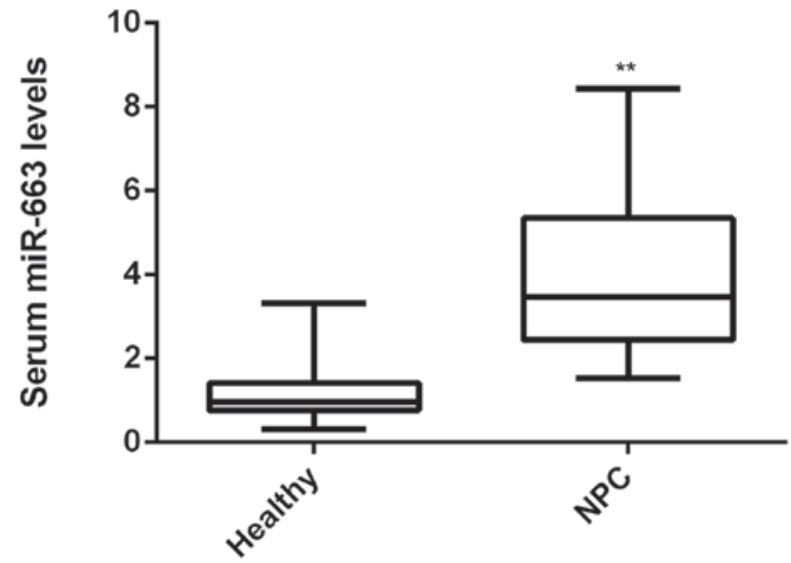

Figure 1. miR-663 expression in patients with NPC. Reverse transcription-quantitative polymerase chain reaction analysis was performed to examine the serum miR-663 levels in patients with NPC $(n=74)$ compared with healthy controls $(n=27) .{ }^{* *} \mathrm{P}<0.01$ vs. healthy. miR, microRNA; NPC, nasopharyngeal carcinoma. The bottom and top of the box plot represent the first and third quartiles, the center line represents the median and the whiskers represent the minimum and maximum of the data.

present study demonstrated that the knockdown of miR-663 decreased the proliferation of C666-1 cells via the induction of cell cycle arrest at the G1 stage.

CDKN2A is a direct target gene of miR-663 in NPC cells. The present study demonstrated that CDKN2A was a predicted target gene of miR-663 using Targetscan software, and that this association was evolutionarily conserved (Fig. 3A). In order to confirm the targeting of CDKN2A by miR-663, luciferase vectors containing the WT or MT CDKN2A 3'-UTR were generated (Fig. 3B and C). A luciferase reporter assay was performed, and it was observed that the luciferase activity was significantly decreased in C666-1 cells co-transfected with the WT-CDKN2A luciferase reporter vector and miR-663 mimics, compared with the control group (Fig. 3D). However, these effects were reversed by transfection with the MT-CDKN2A luciferase reporter vector. Therefore, the results of the present study indicated that miR-663 may directly bind to the 3'UTR of CDKN2A mRNA.

The effects of miR-663 on CDKN2A expression were further examined. Western blotting data demonstrated that the knockdown of miR-663 significantly increased the protein expression of CDKN2A in C666-1 cells (Fig. 4A). In order to further confirm these results, C666-1 cells were transfected with miR-663 mimic, and the miR-663 levels were significantly increased following transfection (Fig. 4B). Overexpression of miR-663 significantly decreased the CDKN2A protein level in C666-1 cells (Fig. 4C). The results of the present study demonstrated that miR-663 negatively-regulated the expression of CDKN2A via direct binding to the 3'UTR of CDKN2A mRNA in C666-1 cells.

CDKN2A is associated with the miR-663-mediated increase in proliferation and the cell cycle progression of NPC cells. It was hypothesized that CDKN2A may be associated with the miR-663-mediated increase in NPC cell viability and with cell cycle progression. C666-1 cells were transfected with a miR-663 inhibitor, or co-transfected with the miR-663 inhibitor and 
Table I. Association between serum miR-663 levels and clinicopathological characteristics of patients with nasopharyngeal carcinoma.

\begin{tabular}{|c|c|c|c|c|}
\hline Variable & No. of patients & Low miR-663 & High miR-663 & P-value \\
\hline Age, years & & & & 0.980 \\
\hline$<55$ & 36 & 20 & 16 & \\
\hline$\geq 55$ & 38 & 21 & 17 & \\
\hline Sex & & & & 0.939 \\
\hline Male & 40 & 22 & 18 & \\
\hline Female & 34 & 19 & 15 & \\
\hline Grade & & & & 0.013 \\
\hline $\mathrm{G} 1 / 2$ & 41 & 28 & 13 & \\
\hline G3 & 33 & 13 & 20 & \\
\hline T stage & & & & 0.112 \\
\hline $\mathrm{T} 1 / 2$ & 39 & 25 & 14 & \\
\hline $\mathrm{T} 3 / 4$ & 35 & 16 & 19 & \\
\hline Lymph node metastasis & & & & 0.001 \\
\hline No & 41 & 0 & 11 & \\
\hline Yes & 33 & & 22 & \\
\hline Distant metastasis & & & & 0.172 \\
\hline No & 53 & 32 & 21 & \\
\hline Yes & 21 & 9 & 12 & \\
\hline Clinical stage & & & & 0.001 \\
\hline $\mathrm{I} / \mathrm{II}$ & 39 & 29 & 10 & \\
\hline III/IV & 35 & 12 & 23 & \\
\hline EBV infection & & & & 0.239 \\
\hline No & & 6 & 2 & \\
\hline Yes & & 35 & 31 & \\
\hline
\end{tabular}

miR, microRNA; T stage, tumor stage, EBV, Epstein-Barr virus.

CDKN2A small interfering (si)RNA. It was observed that the protein expression of CDKN2A was significantly decreased in the miR-663 inhibitor + CDKN2A siRNA group, compared with the miR-663 inhibitor group (Fig. 5A). An MTT assay further demonstrated that the viability of C666-1 cells was significantly decreased in the miR-663 inhibitor + CDKN2A siRNA group, compared with the miR-663 inhibitor group (Fig. 5B), suggesting that inhibition of CDKN2A expression reversed the suppressive effects of miR-663 knockdown on C666-1 cell proliferation. The cell cycle distribution was additionally assessed. As presented in Fig. 5C, a reduced number of C666-1 cells were at the G1 stage in the miR-663 inhibitor + CDKN2A siRNA group compared with the miR-663 inhibitor group, suggesting that the inhibition of CDKN2A reversed the suppressive effect of miR-663 knockdown on cell cycle progression in C666-1 cells. Therefore, CDKN2A is associated with the miR-663-mediated cell cycle progression and proliferation of NPC cells.

CDKN2A is downregulated in NPC cells. The expression of CDKN2A in C666-1 cells was examined, and NP69 cells were used as a control. RT-qPCR analysis and western blotting indicated that the mRNA and protein levels of CDKN2A were significantly decreased in C666-1 cells compared with NP69 cells (Fig. 6A and B). Therefore, the decreased expression of CDKN2A may be caused by the increased expression of miR-663, which promotes NPC cell proliferation.

\section{Discussion}

The regulatory mechanism of miR-663 in NPC cell proliferation remains to be completely elucidated. In the present study, it was observed that miR-663 was significantly upregulated in the serum of patients with NPC, and that the increased serum miR-663 levels were significantly associated with advanced progression of NPC. In addition, miR-663 was demonstrated to be upregulated in the C666-1 NPC cell line. The inhibition miR-663 significantly decreased the proliferation of C666-1 cells by inducing cell cycle arrest at the G1 stage. CDKN2A was identified to be a target gene of miR-663, and its protein expression was negatively regulated by miR-663 in C666-1 cells. CDKN2A was associated with miR-663-mediated NPC cell proliferation and cell cycle progression. Additionally, CDKN2A was significantly downregulated in NPC cells compared with NP69 normal epithelial cells. 
A

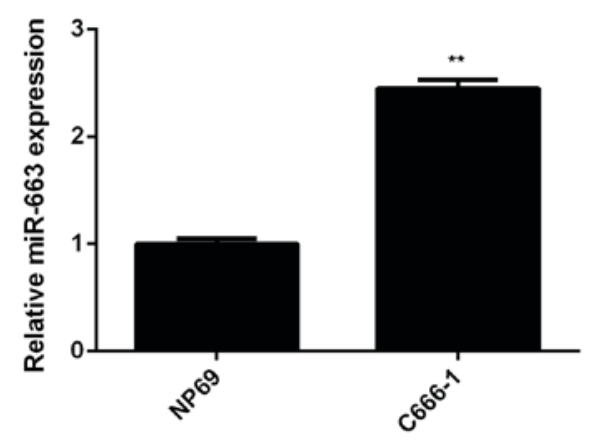

C
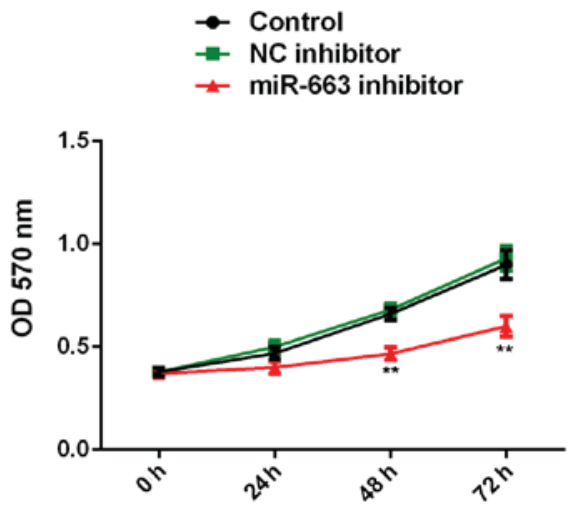

B

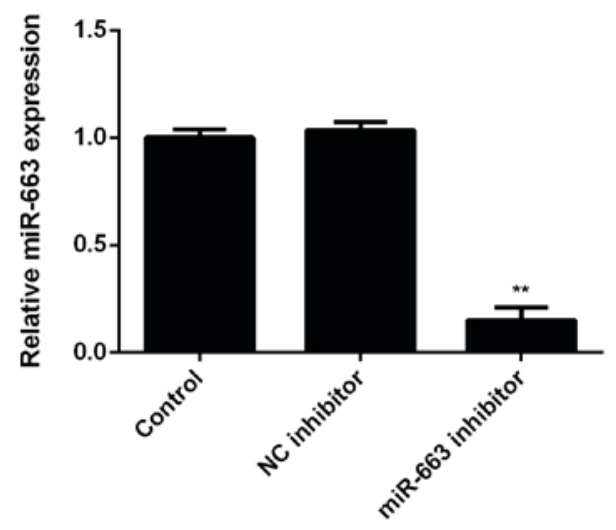

D

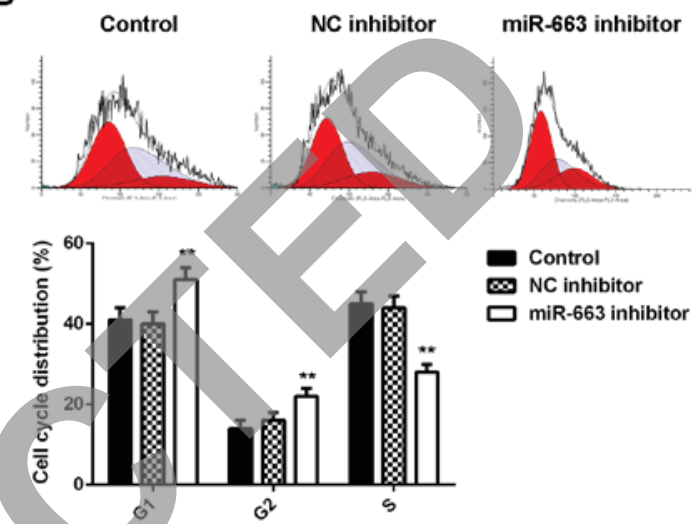

Figure 2. miR-663 expression and cell viability analysis in NPC cells. (A) RT-qPCR analysis was conducted to examine the expression of miR-663 in NPC C666-1 cells compared with normal nasopharyngeal epithelial NP69 cells, " $\mathrm{P}<0.01$ vs. NP69. C666-1 cells were transfected with miR-663 inhibitor or NC inhibitor. (B) RT-qPCR analysis was performed to examine the expression of miR-663. (C) MTT assay and (D) flow cytometry were used to examine the cell viability and cell cycle distribution, respectively. Non-transfected C666-1 cells were used as control. ${ }^{* *} \mathrm{P}<0.01$ vs. control. miR, microRNA; NPC, nasopharyngeal carcinoma; NC, negative control; OD, optical density; RT-qPCR, reverse transcription-quantitative polymerase chain reaction. Data represent the mean of three experimental repeats.

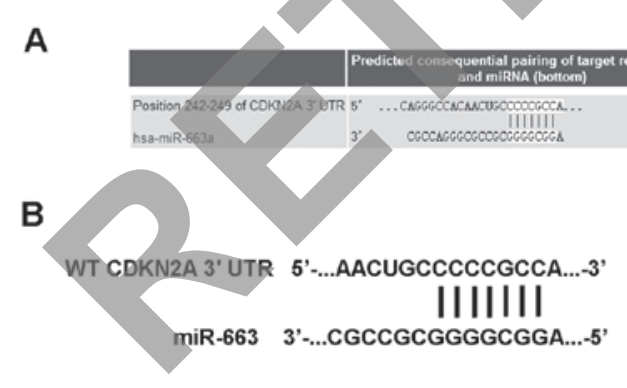

MT CDKN2A 3' UTR 5'-...AACUGCGgGgCGGA...-3'

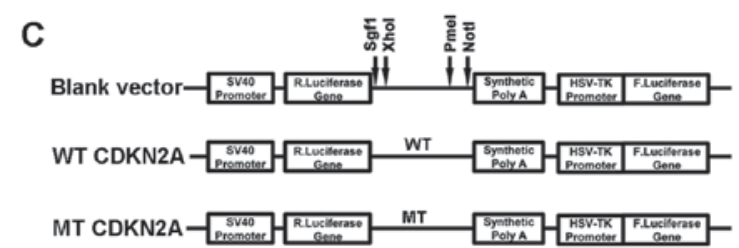

D

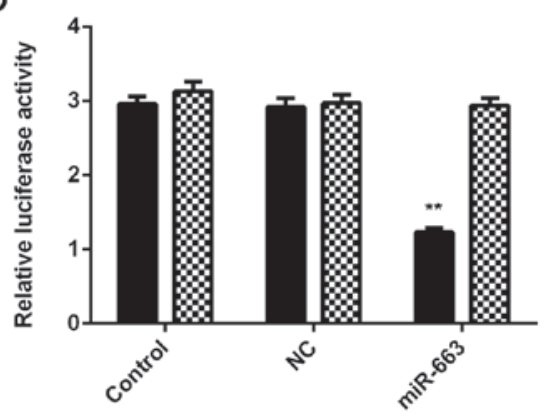

WT CDKN2A 3'UTR - MT CDKN2A 3'UTR

Figure 3. Identification of CDKN2A as a target gene of miR-663. (A) Analysis using Targetscan software indicated that CDKN2A was a target gene of miR-663 and that this association was evolutionally conserved. In order to confirm the targeting of CDKN2A by miR-663, (B) WT or MT CDKN2A 3'-UTRs were generated and inserted into (C) the luciferase reporter vectors. (D) The luciferase activity was significantly reduced in C666-1 cells co-transfected with the WT-CDKN2A luciferase reporter vector and miR-663 mimics, compared with the control group; however, these effects were not observed in cells transfected with the MT-CDKN2A luciferase reporter vector. ${ }^{* *} \mathrm{P}<0.01$ vs. control. miR, microRNA; CDKN2A, cyclin-dependent kinase inhibitor 2A; WT, wild type; MT, mutant; UTR, untranslated region; NC, negative control. Data represent the mean of three experimental repeats. 
A

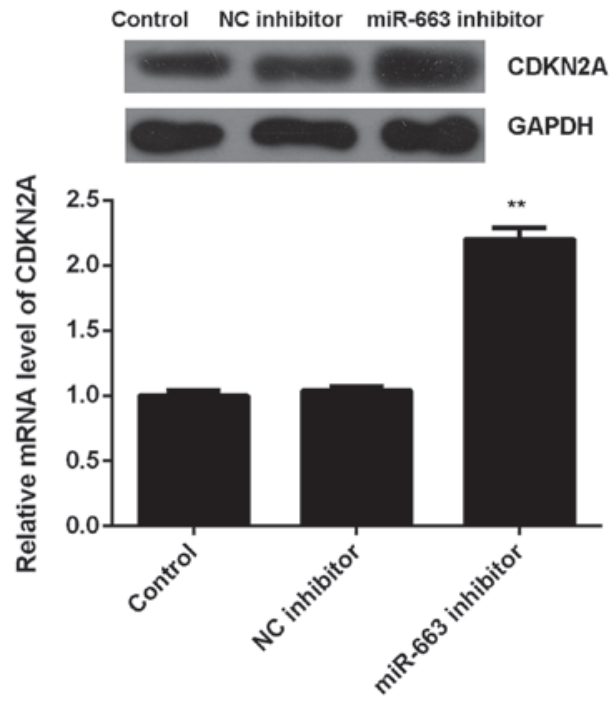

B

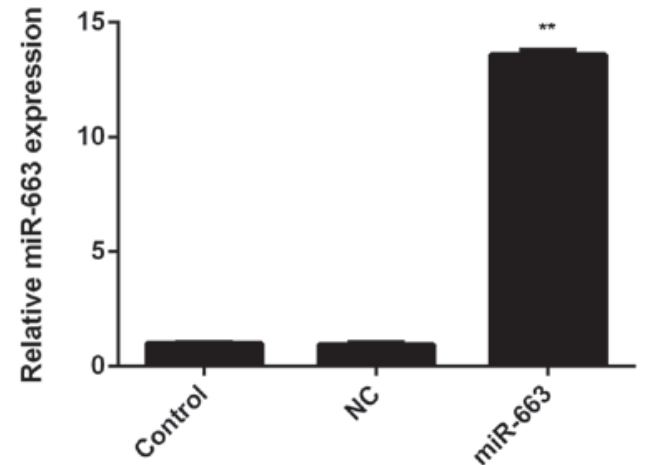

C

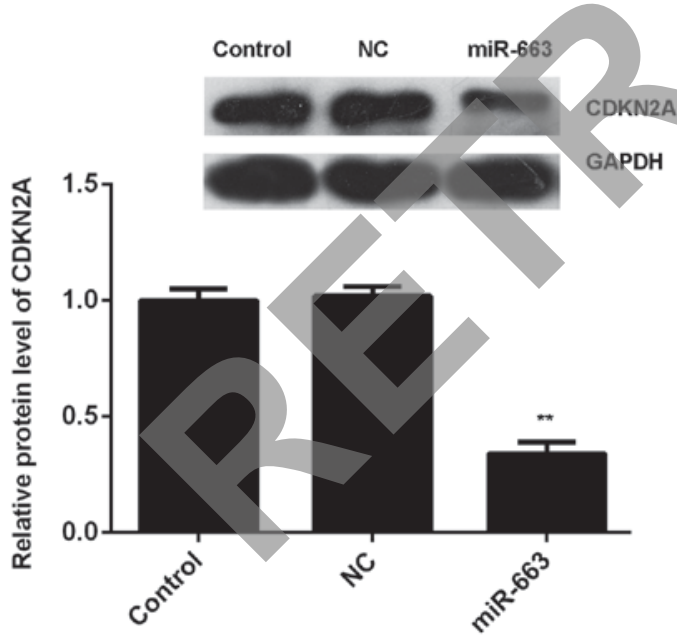

Figure 4. Analysis of the association between CDKN2A and miR-663 expression in nasopharyngeal carcinoma. (A) Western blotting was used to examine the protein expression of CDKN2A in C666-1 cells transfected with miR-663 inhibitor or NC inhibitor. (B) Reverse transcription-quantitative polymerase chain reaction analysis was used to examine the miR-663 levels in C666-1 cells transfected with miR-663 mimic, or scramble miR as the NC group. (C) Western blotting was used to examine the protein expression of CDKN2A. Non-transfected C666-1 cells were used as the control. ${ }^{* *} \mathrm{P}<0.01$ vs. control. miR, microRNA; CDKN2A, cyclin-dependent kinase inhibitor 2A; NC, negative control. Data represent the mean of three experimental repeats.

miR-663 has previously been demonstrated to serve a promotive or tumor suppressive role in a number of types of cancer (13,24-26). For example, Pan et al (13) reported that miR-663 was downregulated in gastric cancer, and that overexpression of miR-663 inhibited the proliferation of gastric cancer cells in vitro and tumor growth in vivo. Tili et al (24) observed that treatment with resveratrol promoted the expression of miR-663, and thereby inhibited the canonical transforming growth factor $\beta$ pathway in SW480 colon cancer cells, suggesting that upregulation of miR-663 may be associated with the anti-cancer effects of resveratrol in colon cancer. Yan-Fang et al (27) demonstrated that miR-663 was significantly downregulated in pediatric acute myeloid leukemia (AML) cells compared with normal bone marrow controls, suggesting that its downregulation may be associated with AML development. By contrast, miR-663 may function as an onco-miR in other types of cancer. miR-633 was observed to be upregulated in malignant melanoma compared with benign melanocytic nevi (25). miR-633 was additionally upregulated in lung cancer, and promoted the proliferation of lung cancer cells by targeting a number of tumor suppressors (26). Therefore, miR-663 serves different roles in different types of cancer, which may be due to varied tumor microenyironments. In the present study, it was observed that serum miR-663 levels were significantly increased in patients with NPC compared with those healthy controls. In addition, the expression of miR-663 was significantly increased in NPC C666-1 cells compared with NP69 cells. Yi et al (15) demonstrated that the expression of miR-663 was significantly increased in NPC tissues and cell lines (15), which is consistent with the findings of the present study. The results of the present study demonstrated that the increased serum levels of miR-663 were significantly associated with grade, lymph node metastasis, and clinical stage, suggesting that upregulation of miR-663 may be associated with NPC progression. It was further demonstrated that the knockdown of miR-663 significantly decreased the proliferation of C666-1 cells via the induction of cell cycle arrest at the G1 stage, suggesting that miR-663 serves a promotive role in NPC cell proliferation. Similarly, Yi et al (15) reported that miR-663 was able to promote the proliferation of NPC cells in vitro and in vivo. It was additionally demonstrated that P21 was associated with miR-663-mediated NPC cell proliferation (15). By directly inhibiting the expression of P21, miR-663 may promote the cellular G1/S transition in NPC cells (15).

As each miR may target a number of genes (7), other targets of miR-663 may serve roles in the regulation of NPC cell proliferation. Therefore, bioinformatic analysis was performed in the present study to predict the putative target genes of miR-663, and a luciferase reporter assay data indicated that miR-663 was able to directly bind to the 3'UTR of CDKN2A mRNA. The protein expression of CDKN2A was negatively regulated by miR-663 in C666-1 cells. Therefore, CDKN2A is a direct target gene of miR-663 in NPC cells. CDKN2A has been demonstrated to be an important tumor suppressor (28). Downregulation of CDKN2A has been suggested to be associated with radioresistance in NPC cells (29). Zhong et al (30) reported that histone-lysine $\mathrm{N}$-methyltransferase $\mathrm{EZH} 2$ may promote the proliferation and cell cycle progression of NPC cells, by inhibiting the expression of CDKN2A via hypermethylation of its promoter. In the present study, it was observed that siRNA knockdown of CDKN2A significantly reversed the effects of the miR-663 inhibitor on C666-1 cell proliferation and cell cycle progression, suggesting that miR-663 promotes 
A
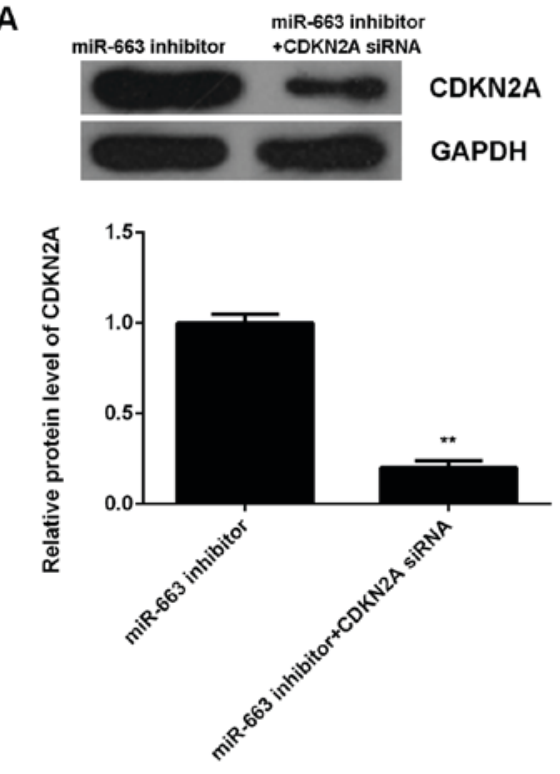

B

\pm miR-663 inhibitor

miR-663 inhibitor+CDKN2A siRNA

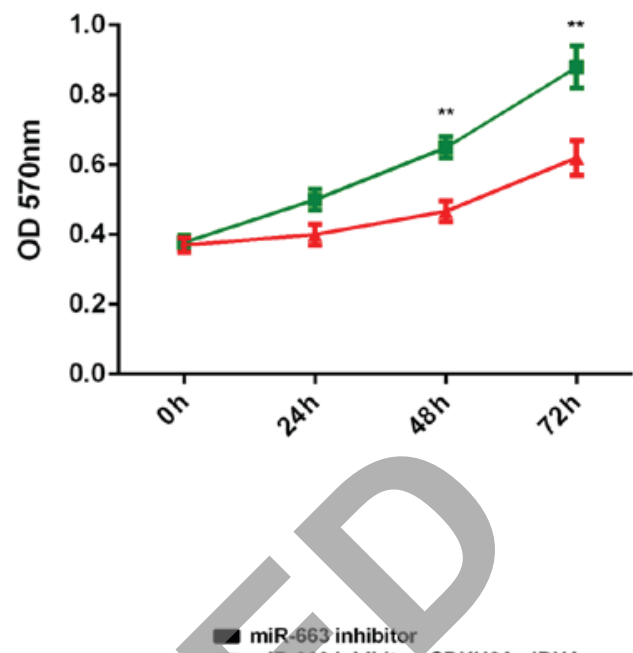

miR-663 inhibitor+CDKN2A siRNA

C

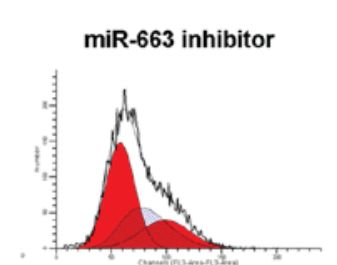

miR-663 inhibitor +CDKN2A siRNA
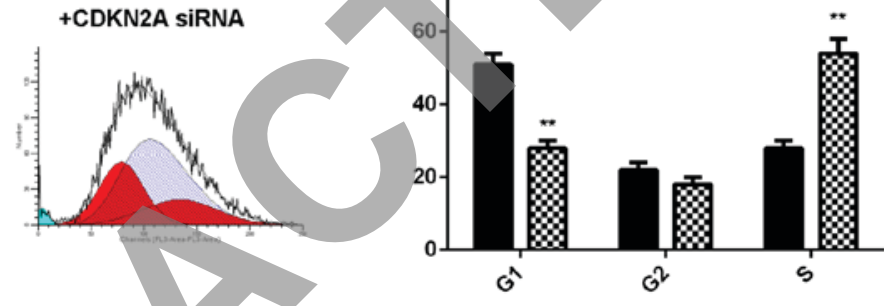

Figure 5. Analysis of the effect of CDKN2A expression on cell viability and cell cycle distribution in nasopharyngeal carcinoma. C666-1 cells were transfected with miR-663 inhibitor, or co-transfected with miR-663 inhibitor and CDKN2A siRNA. (A) Reverse transcription-quantitative polymerase chain reaction analysis was performed to examine the gene expression of CDKN2A. (B) An MTT assay and (C) flow cytometry were used to examine cell viability and the cell cycle distribution. ${ }^{* *} \mathrm{P}<0.01$ vs. miR-663 inhibitor. miR, microRNA; CDKN2A, cyclin-dependent kinase inhibitor 2A; siRNA, small interfering RNA; OD, optical density. Data represent the mean of three experimental repeats.

A

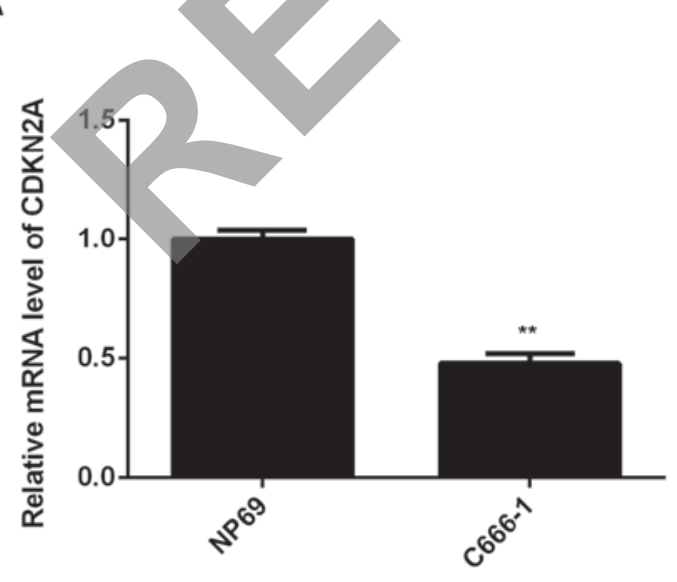

B

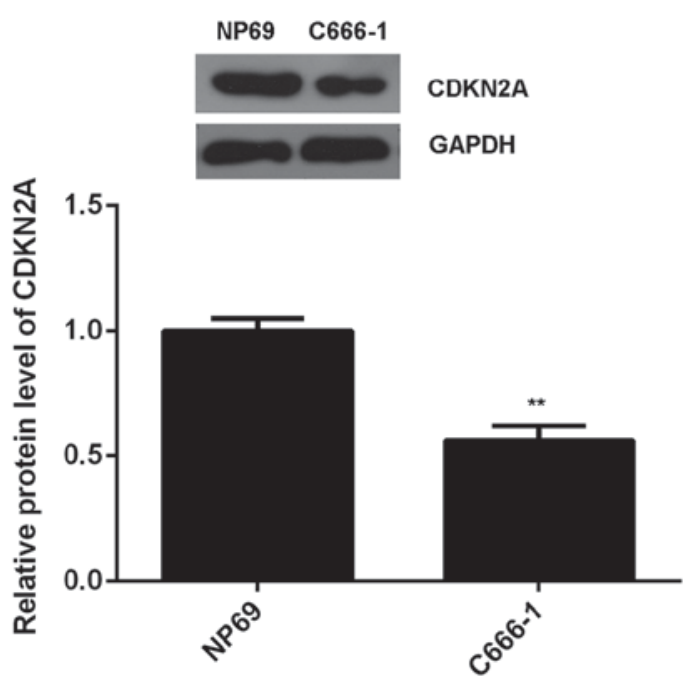

Figure 6. CDKN2A expression in NPC and normal cells. (A) Reverse transcription-quantitative polymerase chain reaction analysis and (B) western blotting were used to examine the mRNA and protein levels of CDKN2A in C666-1 NPC cells, compared with NP69 normal nasopharyngeal epithelial cells. ${ }^{* *} \mathrm{P}<0.01$ vs. NP69. miR, microRNA; CDKN2A, cyclin-dependent kinase inhibitor 2A; NPC, nasopharyngeal carcinoma. Data represent the mean of three experimental repeats.

C666-1 cell proliferation by inhibiting the expression of CDKN2A, and thereby enhancing cell cycle progression.
In addition, it was demonstrated that the protein expression of CDKN2A was significantly decreased in C666-1 cells. 
Similarly, CDKN2A has been reported to be significantly downregulated in NPC tissues, and frequently methylated in the serum of patients with NPC $(31,32)$. The results of the present study suggested that the decreased expression of CDKN2A may be caused by the upregulation of miR-663 in NPC.

In conclusion, miR-663 is upregulated in the serum of patients with NPC and NPC cells, and may promote the proliferation and cell cycle progression of NPC cells, at least in part through direct targeting of CDKN2A. The results of the present study suggested that miR-663 may be used as a therapeutic target for NPC.

\section{Acknowledgements}

The present study was supported by the Special Fund Project for Technology Innovation of Foshan City (grant no. 2014AG10003).

\section{References}

1. Wei WI and Sham JS: Nasopharyngeal carcinoma. Lancet 365: 2041-2054, 2005.

2. Yip TT, Ngan RK, Fong AH and Law SC: Application of circulating plasma/serum EBV DNA in the clinical management of nasopharyngeal carcinoma. Oral Oncol 50: 527-538, 2014.

3. Sze H, Blanchard P, Ng WT, Pignon JP and Lee AW: Chemotherapy for nasopharyngeal carcinoma-current recommendation and controversies. Hematol Oncol Clin North Am 29: 1107-1122, 2015.

4. Xiao L, Xiao T, Wang ZM, Cho WC and Xiao ZQ: Biomarker discovery of nasopharyngeal carcinoma by proteomics. Expert Rev Proteomics 11: 215-225, 2014.

5. Lee AW, Lin JC and Ng WT: Current management of nasopharyngeal cancer. Semin Radiat Oncol 22; 233-244,2012.

6. Yates LA, Norbury CJ and Gilbert RJ; The long and short of microRNA. Cell 153: 516-519, 2013.

7. Ambros V: The functions of animal microRNAs. Nature 431: 350-355, 2004.

8. Calin GA and Croce CM: MícroRNA signatures in human cancers. Nat Rev Cancer 6: 857-866, 2006.

9. Sidhu K, Kapoor NR, Pandey V and Kumar V: The 'Macro' World of microRNAs in hepatocellular carcinoma. Front Oncol 5: 68, 2015.

10. Hung $\mathrm{CH}$, Chiu $\mathrm{YC}$, Chen $\mathrm{CH}$ and $\mathrm{Hu} \mathrm{TH}$ : MicroRNAs in hepatocellular carcinoma: Carcinogenesis, progression, and therapeutic target. Biomed Res Int 2014: 486407, 2014.

11. Alajez NM, Lenarduzzi M, Ito E, Hui AB, Shi W, Bruce J, Yue S, Huang SH, Xu W, Waldron J, et al: miR-218 suppresses nasopharyngeal cancer progression through downregulation of survivin and the SLIT2-ROBO1 pathway. Cancer Res 71: 2381-2391, 2011

12. Deng M, Ye Q, Qin Z, Zheng Y, He W, Tang H, Zhou Y, Xiong W, Zhou M, Li X, et al: miR-214 promotes tumorigenesis by targeting lactotransferrin in nasopharyngeal carcinoma. Tumour Biol 34: 1793-1800, 2013.

13. Pan J, Hu H, Zhou Z, Sun L, Peng L, Yu L, Sun L, Liu J, Yang Z and Ran Y: Tumor-suppressive mir-663 gene induces mitotic catastrophe growth arrest in human gastric cancer cells. Oncol Rep 24: 105-112, 2010.
14. Jiao L, Deng Z, Xu C, Yu Y, Li Y, Yang C, Chen J, Liu Z, Huang G, Li LC and Sun Y: miR-663 induces castration-resistant prostate cancer transformation and predicts clinical recurrence. J Cell Physiol 229: 834-844, 2014.

15. Yi C, Wang Q, Wang L, Huang Y, Li L, Liu L, Zhou X, Xie G, Kang $\mathrm{T}$, Wang $\mathrm{H}$, et al: MiR-663, a microRNA targeting $\mathrm{p} 21$ (WAF1/CIP1), promotes the proliferation and tumorigenesis of nasopharyngeal carcinoma. Oncogene 31: 4421-4433, 2012.

16. Ruas M and Peters G: The p16INK4a/CDKN2A tumor suppressor and its relatives. Biochim Biophys Acta 1378: F115-F177, 1998.

17. Aoude LG, Wadt KA, Pritchard AL and Hayward NK: Genetics of familial melanoma: 20 years after CDKN2A. Pigment Cell Melanoma Res 28: 148-160, 2015.

18. Williams RT and Sherr CJ: The INK4-ARF (CDKN2A/B) locus in hematopoiesis and BCR-ABL-induced leukemias. Cold Spring Harb Symp Quant Biol 73: 461-467, 2008.

19. Xing X, Cai W, Shi H, Wang Y, Li M, Jiao J and Chen M: The prognostic value of CDKN2A hypermethylation in colorectal cancer: A meta-analysis. Br J Cancer 108: 2542-2548, 2013.

20. Jiang W, Chamberlain PD, Garden AS, Kim BY, Ma D, Lo EJ, Bell D, Gunn GB, Fuller CD, Rosenthal DI, et al: Prognostic value of p16 expression in Epstein-Barr virus-positive nasopharyngeal carcinomas. Head Neck 38 (Suppl 1): E1459-E1466, 2016.

21. Shao Y, Jiang H, Wu X, Luo Y and Tang W: p16 promoter hypermethylation is associated with increased risk of nasopharyngeal carcinoma. Mol Clin Oncol 2: 1121-1124, 2014.

22. Zong J, Huang Q, Guo Q and Pan J: Evolution of the Chinese staging system for nasopharyngeal carcinoma. Chin Clin Oneol 5: 19, 2016

23. Livak KJ and Schmittgen TD: Analysis of relative gene expression data using real-time quantitative PCR and the 2(-Delta Delta C(T)) method. Methods 25: 402-408, 2001.

24. Tili E, Michaille JJ, Alder H, Volinia S, Delmas D, Latruffe N and Croce CM: Resveratrol modulates the levels of microRNAs targeting genes encoding tumor-suppressors and effectors of TGF $\beta$ signaling pathway in SW480 cells. Biochem Pharmacol 80: 2057-2065, 2010

5. Sand M, Skrygan M, Sand D, Georgas D, Gambichler T, Hahn SA, Altmeyer P and Bechara FG: Comparative microarray analysis of microRNA expression profiles in primary cutaneous malignant melanoma, cutaneous malignant melanoma metastases and benign melanocytic nevi. Cell Tissue Res 351: 85-98, 2013.

26. Liu ZY, Zhang GL, Wang MM, Xiong YN and Cui HQ: MicroRNA-663 targets TGFB1 and regulates lung cancer proliferation. Asian Pac J Cancer Prev 12: 2819-2823, 2011.

27. Yan-Fang T, Jian N, Jun L, Na W, Pei-Fang X, Wen-Li Z, Dong W, Li P, Jian W, Xing F and Jian P: The promoter of miR-663 is hypermethylated in Chinese pediatric acute myeloid leukemia (AML). BMC Med Genet 14: 74, 2013

28. Kotake Y, Naemura M, Murasaki C, Inoue Y and Okamoto H: Transcriptional regulation of the p16 tumor suppressor gene. Anticancer Res 35: 4397-4401, 2015.

29. Peng G, Cao RB, Li YH, Zou ZW, Huang J and Ding Q: Alterations of cell cycle control proteins SHP1/2, p16, CDK4 and cyclin D1 in radioresistant nasopharyngeal carcinoma cells. Mol Med Rep 10: 1709-1716, 2014.

30. Zhong J, Min L, Huang H, Li L, Li D, Li J, Ma Z and Dai L: EZH2 regulates the expression of p16 in the nasopharyngeal cancer cells. Technol Cancer Res Treat 12: 269-274, 2013.

31. Huang $\mathrm{HH}$, Chen $\mathrm{CH}$, Huang $\mathrm{SC}$, Yang $\mathrm{CH}$ and Hwang $\mathrm{CF}$ : Expression of 14-3-3 sigma, cyclin-dependent kinases 2 and 4, p16, and Epstein-Barr nuclear antigen 1 in nasopharyngeal carcinoma. J Laryngol Otol 128: 134-141, 2014.

32. Tian F, Yip SP, Kwong DL, Lin Z, Yang Z and Wu VW: Promoter hypermethylation of tumor suppressor genes in serum as potential biomarker for the diagnosis of nasopharyngeal carcinoma. Cancer Epidemiol 37: 708-713, 2013. 